\title{
Maternal alcohol consumption and offspring DNA methylation: findings from six general population-based birth cohorts
}

\author{
Gemma C Sharp ${ }^{\ddagger 1,2,3}$, Ryan Arathimos ${ }^{*} \ddagger 1,2$, Sarah E Reese ${ }^{4}$, Christian M Page ${ }^{5,6}$, Janine \\ Felix $^{7,8,9}$, Leanne K Küpers ${ }^{1,2,10}$, Sheryl L Rifas-Shiman ${ }^{11}$, Chunyu Liu ${ }^{12,13,14}$, The Cohorts for \\ Heart and Aging Research in Genomic Epidemiology plus (CHARGE + ) methylation alcohol \\ working group, Kimberley Burrows ${ }^{1,2}$, Shanshan Zhao ${ }^{4}$, Maria C Magnus ${ }^{1,2,5}$, Liesbeth \\ Duijts $^{7,15,16}$, Eva Corpeleijn ${ }^{10}$, Dawn L DeMeo ${ }^{17}$, Augusto Litonjua ${ }^{17}$, Andrea Baccarelli ${ }^{18}$, \\ Marie-France Hivert ${ }^{11,19}$, Emily Oken ${ }^{11}$, Harold Snieder ${ }^{10}$, Vincent Jaddoe ${ }^{7,8,9}$, Wenche \\ Nystad $^{5}$, Stephanie J London ${ }^{4}$, Caroline L Relton ${ }^{1,2}$ \& Luisa Zuccolo ${ }^{1,2}$ \\ ${ }^{1} \mathrm{MRC}$ Integrative Epidemiology Unit, University of Bristol, Bristol, BS8 2BN, UK \\ ${ }^{2}$ School of Social \& Community Medicine, University of Bristol, Bristol, BS8 2BN, UK \\ ${ }^{3}$ School of Oral \& Dental Sciences, University of Bristol, Bristol, UK \\ ${ }^{4}$ Division of Intramural Research, Department of Health \& Human Services, National Institute of Environmental Health Sciences, \\ National Institutes of Health, Research Triangle Park, NC, USA \\ ${ }^{5}$ Division for Mental \& Physical Health, Department of Non-Communicable Diseases, Norwegian Institute of Public Health, Oslo, \\ Norway \\ ${ }^{6}$ Oslo Centre for Biostatistics \& Epidemiology, Oslo University Hospital, Oslo, Norway \\ ${ }^{7}$ The Generation R Study Group, Erasmus MC, University Medical Centre Rotterdam, Rotterdam, The Netherlands \\ ${ }^{8}$ Department of Epidemiology, Erasmus MC, University Medical Centre Rotterdam, Rotterdam, The Netherlands \\ ${ }^{9}$ Department of Pediatrics, Erasmus MC, University Medical Centre Rotterdam, Rotterdam, The Netherlands \\ ${ }^{10}$ Department of Epidemiology, University of Groningen, University Medical Center Groningen, Groningen, The Netherlands \\ ${ }^{11}$ Department of Population Medicine, Harvard Medical School, Harvard Pilgrim Health Care Institute, Boston, MA, USA \\ ${ }^{12}$ The Framingham Heart Study, Framingham, MA, USA \\ ${ }^{13}$ The Population Sciences Branch, Division of Intramural Research, National Heart, Lung, \& Blood Institute, Bethesda, MD, USA \\ ${ }^{14}$ Department of Biostatistics, Boston University School of Public Health, 715 Albany St, Boston, MA, USA \\ ${ }^{15}$ Department of Pediatrics, Division of Respiratory Medicine \& Allergology, Erasmus MC, University Medical Centre Rotterdam, \\ Rotterdam, The Netherlands \\ ${ }^{16}$ Department of Pediatrics, Division of Neonatology, Erasmus MC, University Medical Centre Rotterdam, Rotterdam, The \\ Netherlands \\ ${ }^{17}$ Channing Division of Network Medicine, Brigham \& Women's Hospital, Harvard Medical School, Boston, MA, USA \\ ${ }^{18}$ Laboratory of Precision Environmental Biosciences, Columbia University Mailman School of Public Health, New York, NY, USA \\ ${ }^{19}$ Diabetes Unit, Massachusetts General Hospital, Boston, MA, USA \\ * Author for correspondence: ryan.arathimos@bristol.ac.uk \\ ${ }_{\ddagger}^{\ddagger}$ Authors contributed equally
}

\begin{abstract}
Aim: Alcohol consumption during pregnancy is sometimes associated with adverse outcomes in offspring, potentially mediated by epigenetic modifications. We aimed to investigate genome-wide DNA methylation in cord blood of newborns exposed to alcohol in utero. Materials \& methods: We meta-analyzed information from six population-based birth cohorts within the Pregnancy and Childhood Epigenetics consortium. Results: We found no strong evidence of association at either individual $\mathrm{CpGs}$ or across larger regions of the genome. Conclusion: Our findings suggest no association between maternal alcohol consumption and offspring cord blood DNA methylation. This is in stark contrast to the multiple strong associations previous studies have found for maternal smoking, which is similarly socially patterned. However, it is possible that a combination of a larger sample size, higher doses, different timings of exposure, exploration of a different tissue and a more global assessment of genomic DNA methylation might show evidence of association.
\end{abstract}

First draft submitted: 3 August 2017; Accepted for publication: 27 September 2017; Published online: 27 November 2017

Keywords: alcohol • cord blood • DNA methylation • epidemiology • epigenetics • meta-analysis • PACE consortium - pregnancy 
It is well known that heavy alcohol consumption during pregnancy can cause Fetal Alcohol Spectrum Disorders (FASD), a spectrum of disorders characterized by a continuum of structural and neurodevelopmental abnormalities, with Fetal Alcohol Syndrome at the more severe end of the spectrum [1-3]. The severity of FASD appears to depend largely on the timing, dose and frequency of exposure to alcohol, with heavy exposure in the latter half of the first trimester being associated with the most severe effect [4,5]. However, in the general population most pregnant women do not drink at the doses required to cause FASD. For example, in population-based studies from Ireland, the UK, Australia and New Zealand, around 70\% (range: 67-77\%) of women who reported drinking in the first trimester consumed seven units or fewer per week, which is considered light-to-moderate consumption. In the second trimester, nearly all women who drank (range 99-100\%) consumed seven units or fewer per week [6]. Evidence of an effect of light-to-moderate levels of prenatal alcohol exposure is sparse and inconsistent. Although the majority of systematic reviews and studies published after these reviews have not found convincing evidence of association between light-to-moderate drinking and adverse offspring health and neurodevelopment [7-14], a recent comprehensive review of prospective studies found suggestive evidence of an association between mothers consuming up to four UK units of alcohol per week and babies born small-for-gestational age or preterm [15]. Furthermore, results from quasi-experimental study designs, which are more robust to the presence of confounding by parental socio-economic factors, have shown some evidence of effect of (mostly light-to-moderate) maternal alcohol consumption on offspring cognition and behavior [16-18]. The inconsistency of findings may be explained by the failure to adequately control for certain confounding factors, such as socioeconomic position, diet and ethnicity [19], which affect offspring outcomes both prenatally and postnatally, and could therefore bias any potentially small effect of light-to-moderate drinking in pregnancy [20]. Suggestions of harm from these later studies, together with findings from animal experiments, have prompted the UK Chief Medical Officer to recently revise the guidelines for alcohol drinking in pregnancy to recommend abstention, based on the precautionary principle [21].

Whether there is a causal association between light-to-moderate drinking in pregnancy and children's health outcomes is an important question. Identifying a possible biological pathway showing effects at birth would be a first step toward providing an answer. Currently, precise biological mechanisms underlying potential adverse effects of prenatal alcohol exposure are unknown. However, epigenetic modifications have been suggested as one such potential mediator, with some evidence that this is the case for prenatal exposure to smoking [22-24].

Animal studies suggest that alcohol exposure affects DNA methylation levels both globally, through its antagonistic effect on methyl donors such as folate [25,26], and in a gene-specific fashion [27,28]. Mouse pups exposed to alcohol during the highly epigenetically-sensitive intrauterine period show dose- and timing-specific epigenetic effects, including DNA methylation effects that correlate with long-lasting changes in gene expression and could potentially drive offspring adverse outcomes [29].

Similar experiments are impossible in humans for obvious ethical reasons. However, there is some evidence that treatment with a low physiologically relevant dose of ethanol induces genome-wide changes in DNA methylation in human embryonic stem cells [30]. In addition, a recent observational study of 110 children with FASD and 96 controls found genome-wide differences in buccal epithelial cell DNA methylation [31]. It is still unknown whether light-to-moderate prenatal alcohol exposure is associated with differential DNA methylation in human offspring.

In this study, we meta-analyzed epigenome-wide association study (EWAS) summary statistics from six population-based cohort studies within the Pregnancy and Childhood Epigenetics (PACE) Consortium to investigate DNA methylation profiles in the cord blood of newborns differentially exposed to alcohol in utero. We also compared these associations with those recently found in studies of differential buccal cell DNA methylation in children with FASD compared with controls [31], and differential whole blood DNA methylation in adults in the general population who drink light-to-moderately compared with adults who do not drink [32].

\section{Materials \& methods}

\section{Participating cohorts}

A total of six independent cohorts from four countries participated in this study, all were members of the PACE Consortium. Detailed methods for each cohort are provided in the Supplementary Material (Supplementary File 1). All cohorts had data on maternal alcohol consumption before and/or during pregnancy and DNA methylation data as measured using the Illumina Infinium HumanMethylation450k BeadChip array [33]. In alphabetical order, 
these cohorts were: The Avon Longitudinal Study of Parents and Children (ALSPAC) [34-37] from the UK, Groningen Expert Center for Kids with Obesity (GECKO) [38] and Generation R [39,40] from The Netherlands, two independent datasets from the Norwegian Mother and Child Cohort Study (MoBa1, MoBa2) [41,42] and Project Viva (Viva) from the USA [43].

\section{Maternal alcohol consumption (exposure)}

Cohorts assessed maternal alcohol consumption before and during pregnancy via questionnaires completed by the mothers during pregnancy. We were primarily interested in the effects of sustained consumption throughout pregnancy, which represents a longer prenatal exposure to alcohol, potentially interfering with all stages of embryonic and fetal development. Therefore, our main exposure of interest was a binary variable comparing offspring of mothers who drank both before pregnancy and in the second and/or third trimester of pregnancy to offspring of mothers who consumed alcohol before pregnancy but not during the second and/or third trimester of pregnancy. Using this definition, we hoped to compare offspring of mothers who continued to drink alcohol after finding out they were pregnant to offspring of mothers who stopped drinking. From previous research we know that women who drink in the second trimester tend to do so at light-to-moderate levels [6].

Cohorts ran secondary models assessing binge drinking during pregnancy and timing-specific alcohol consumption: before pregnancy, during the first trimester and during the second and/or third trimester. Binge drinking was defined as four, five or six (depending on the cohort) or more glasses per occasion at least once at any time point in pregnancy compared with consuming alcohol before pregnancy and drinking in moderation (i.e., no binge drinking) during pregnancy. Alcohol consumption before pregnancy, during the first trimester and during the second and/or third trimester were all defined using four categories of exposure: no drinking, less than one glass per week, one to six glasses per week and seven or more glasses per week.

\section{Covariates}

All models were adjusted for the potential confounders maternal age (years), maternal education (variable defined by each individual cohort) and maternal smoking status (the preferred categorization was into three groups: no smoking in pregnancy, stopped smoking in early pregnancy, smoking throughout pregnancy. A binary categorization of any versus no smoking was also acceptable). All cohorts also adjusted for technical covariates either by including a batch variable (e.g., chip ID) as a model covariate or by generating and adjusting for surrogate variables. All models were run with and without adjustment for cell counts, which were estimated using the Houseman method [43]. The analyzes were completed before a cord blood reference panel was widely available, so cohorts used an adult whole blood reference [44] to estimate the proportion of B cells, $\mathrm{CD}^{+} \mathrm{T}$ cells, $\mathrm{CD} 4^{+} \mathrm{T}$ cells, granulocytes, NK cells and monocytes in each sample.

\section{Methylation measurements (outcome)}

DNA from cord blood underwent bisulfite conversion using the EZ-96 DNA methylation kit (Zymo Research Corporation, CA, USA). DNA methylation was measured using the Illumina Infinium HumanMethylation450k BeadChip assay at Illumina or in cohort-specific laboratories. Each cohort conducted its own quality control and normalization of methylation data, as detailed in the Supplementary Material (Supplementary File 1). In all analyzes, cohorts used normalized, untransformed $\beta$-values. As a consortium, we have found that extreme outliers in methylation data, likely caused by technical error or rare genetic variants, can have a large influence on results. Therefore, potential outliers were removed. Such outliers were defined using the Tukey method [45], in which an outlier is any value less than the lower quartile minus three-times the interquartile range, or more than the upper quartile plus three-times the interquartile range. This method is appropriate as it is not dependent on distributional assumptions of the data.

\section{Cohort-specific statistical analysis}

Each cohort performed independent EWAS according to a common, prespecified analysis plan. Full EWAS were conducted for each of the alcohol exposures (i.e., sustained alcohol consumption, binge drinking, drinking before pregnancy, drinking in the first trimester, drinking in the second/third trimester). Models were run using multiple robust linear regression (rlm in the MASS R package [46]) in an attempt to control for potential heteroscedasticity in the methylation data. Alcohol consumption was modelled as the exposure and cord blood DNA methylation was the outcome, with adjustment for covariates (and estimated cell counts). 


\section{Meta-analysis}

We performed fixed-effects meta-analysis weighted by the inverse of the variance with METAL [47]. We then excluded control probes $(n=65)$ and probes mapped to the $X(n=11,232)$ or $Y(n=416)$ chromosomes. This left a total of 473,864 probes. Multiple testing was accounted for by controlling the false discovery rate (FDR) at 5\% using the method by Benjamini and Hochberg [48]. Probes were annotated according to hg19 using the IlluminaHumanMethylation450kanno.ilmn12.hg19 R package [49]. We used these annotations to assess enrichment in certain genomic features (relation to CpG island and genomic region) using Fisher's tests.

\section{Sensitivity analyzes}

For the top 500 sites with the smallest p-values in our main model (sustained drinking), we repeated the meta-analysis using a random effects model, to allow for potential differences in effect sizes between cohorts. We additionally assessed interstudy heterogeneity and influence of individual cohorts by observing forest plots and heterogeneity statistics, as well as conducting a 'leave-one-out' analysis using the metafor R package [50]. We checked consistency between models by comparing effect estimates and top hits to those of our primary model. We also compared top hits to a list of probes suggested to give spurious readings due to cross-hybridization or genomic features such as nearby SNPs [51]. When a cord blood reference became available [52], we ran a sensitivity analysis in ALSPAC adjusting for estimated proportions of B-cells, $\mathrm{CD}^{+} \mathrm{T}$ cells, $\mathrm{CD} 4^{+} \mathrm{T}$ cells, granulocytes, NK-cells, monocytes and nucleated red blood cells.

\section{Comparison to associations between FASD \& buccal epithelial DNA methylation in children} We performed a look-up in our results of the top CpGs and differentially methylated regions (DMRs; FDR-adjusted p-value $<0.05$, n CpGs $=658$ ) reported in a study by Portales-Casamar et al. [31], which analyzed epigenome-wide buccal epithelial cell DNA methylation in children with FASD compared with controls. We compared direction of effect and p-values across the two studies.

\section{Comparison to associations between light-to-moderate drinking \& whole blood DNA methylation in adults}

We performed a second look-up in our results of the top CpGs (FDR-adjusted p-value $<0.05$ ) from a study by Liu et al. [32], which meta-analyzed associations between alcohol consumption and epigenome-wide whole blood DNA methylation in adults (CHARGE consortium). In order to harmonize with our models, we restricted the look-up to the top CpGs from the previous study's models that assessed light drinking (three CpGs) and moderate drinking (24 CpGs), versus no drinking (results supplied by the study authors). As with our first look-up, we compared direction of effect and p-values across the two studies.

\section{DMRs analysis}

Adjacent probes on the $450 \mathrm{k}$ array are often highly correlated and DMRs may be more biologically important than individual CpGs. However, there is currently no agreed 'gold-standard' method to identify DMRs and the currently available methods test slightly different (but not necessarily competing) hypotheses. Therefore, to provide confidence that any findings are robust to DMR method, we used two methods, Comb-P [53] and DMRcate [54], to identify DMRs in our meta-analyzed single-CpG EWAS results. Comb-P identifies genomic regions enriched for low p-values, corrects for auto-correlation with neighboring CpGs within 1000 bp using the Stouffer-Liptak method, then adjusts for multiple testing using the Sidak correction. DMRcate generates two smoothed estimates for each chromosome: one weighted by F-statistics (calculated from the meta-analysis results as $[\beta / \text { standard error }]^{2}$ ) and one not, for null comparison. The two estimates are compared via a Satterthwaite approximation and $\mathrm{p}$-values are calculated and adjusted for multiple testing using the FDR method. Regions are defined from groups of significant probes $(\mathrm{FDR}<0.05$ ) where the distance to the next consecutive probe is less than $1000 \mathrm{bp}$. A regional $\mathrm{p}$-value is calculated using the Stouffer method. As a sensitivity analysis, we repeated the Comb-P and DMRcate analyzes using a $500 \mathrm{bp}$ (rather than $1000 \mathrm{bp}$ ) window to define neighboring CpGs.

\section{Blood/brain comparison}

To assess whether identified associations between prenatal alcohol exposure and cord blood methylation are likely to represent associations in a more biologically relevant tissue (the brain), we performed a look-up of CpG sites in a database of correlations between blood and brain methylation [55]. Methods used to derive this database are 


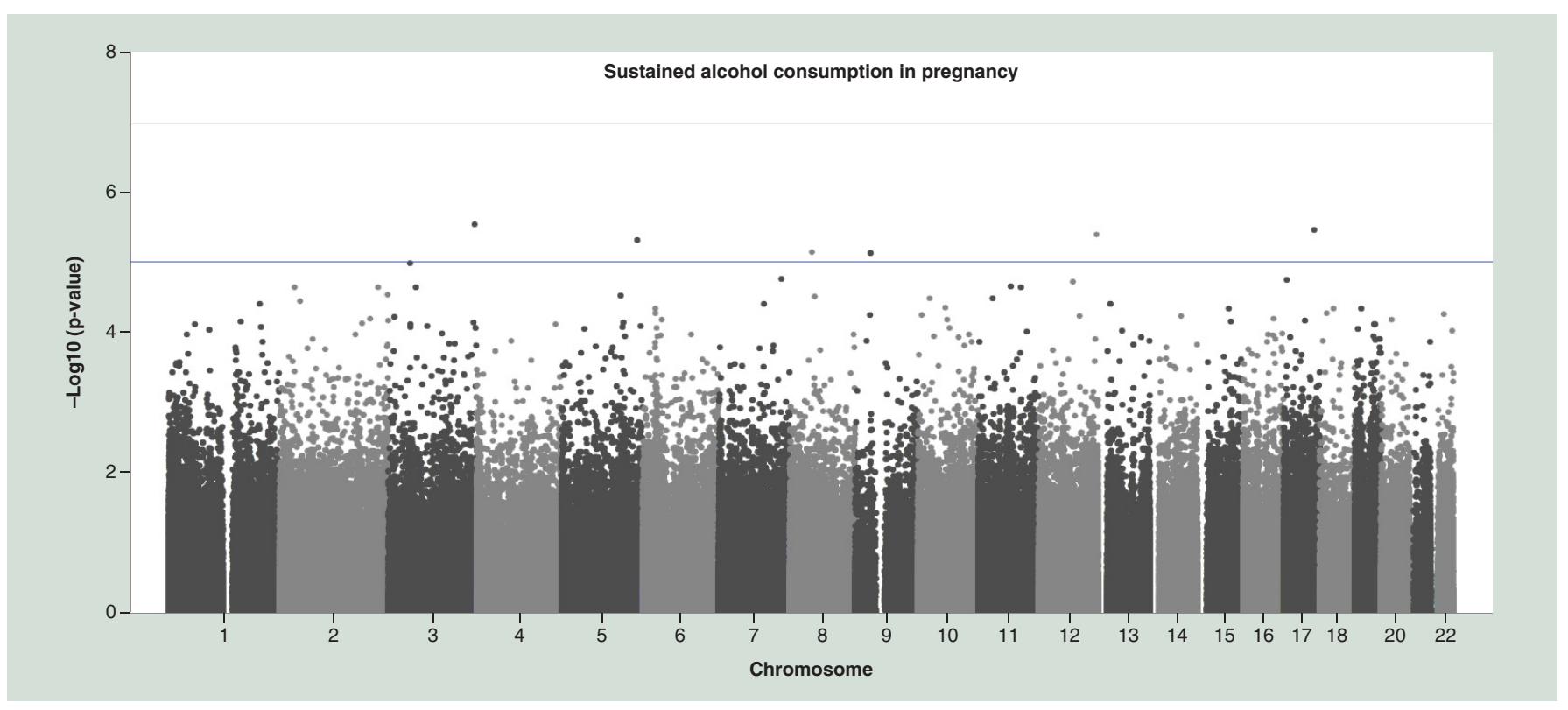

Figure 1. Manhattan plot of sustained alcohol consumption (without adjustment for cell counts).

described elsewhere [56], but briefly, the authors quantified DNA methylation in matched DNA samples from whole blood and four brain regions (prefrontal cortex, entorhinal cortex, superior temporal gyrus and cerebellum) from 122 individuals.

\section{Availability of data}

Data supporting the results reported in this article can be found in the Supplementary Materials. We regret that we are unable to make individual level data available due to concerns regarding compromising individual privacy. However, full meta-analysis results are available from the corresponding author on request.

\section{Results}

\section{Study characteristics}

Of the six participating cohorts, Project Viva and GECKO could not take part in all meta-analyzes due to insufficient data/number of exposed individuals. Project Viva was therefore only included in the analysis of drinking before pregnancy compared with abstaining. GECKO was included in the primary analysis assessing sustained alcohol consumption, and the secondary analysis assessing binge drinking. The other cohorts (ALSPAC, Generation R, MoBa1 and MoBa2) were included in all analyzes.

Five cohorts (ALSPAC, GECKO, Generation R, MoBa1, MoBa2) had the necessary data to take part in our primary analysis of sustained alcohol consumption in pregnancy. The meta-analysis included 3075 mother-child pairs, of which 1147 (37.3\%) mothers consumed alcohol both before and throughout pregnancy and the remaining 1928 mothers consumed alcohol before pregnancy/during the first trimester but not during the second and/or third trimester. Table 1 summarizes the characteristics of each cohort. In all investigated cohorts, women who drank throughout pregnancy were, on average, older and had a higher level of education than women who stopped. In $\mathrm{MoBa} 2$ and GECKO women who drank throughout pregnancy were less likely to smoke throughout pregnancy compared with women who stopped drinking, but the opposite was true for the other three studies.

\section{Primary models: sustained maternal alcohol consumption during pregnancy}

For both the cell-adjusted and cell-unadjusted models, effect sizes were moderate: for top CpGs with p-value $<1$ $\times 10^{-3}$, estimates ranged from a $4 \%$ decrease to $2 \%$ increase in average methylation level in the exposed compared with the unexposed group, with a median absolute estimate of $0.4 \%$ (Supplementary File 2, Supplementary Tables $1 \& 2$ ). No CpG sites survived correction for multiple testing with an FDR-adjusted p-value $<0.05$ (Figure 1). There was little evidence of inflation, as assessed by the lambda value (Table 1). Results (including effect sizes) for all sites with a p-value $<1 \times 10^{-3}$ are presented in Supplementary File 2, Supplementary Tables $1 \& 2$. 


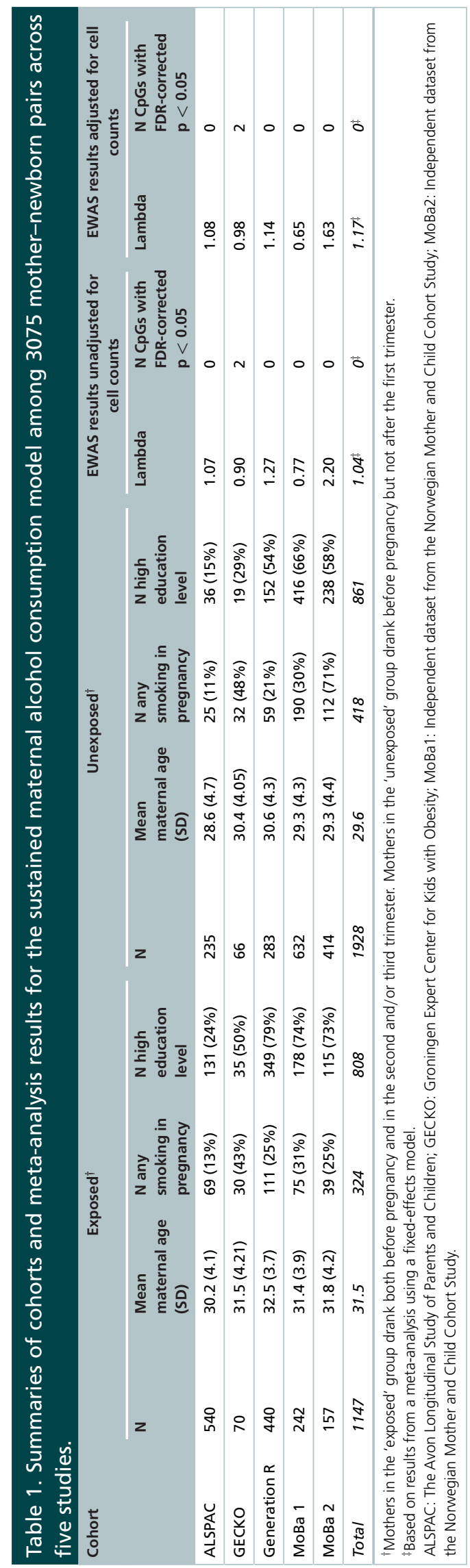


Of the 622 and $797 \mathrm{CpG}$ with p-value $<1 \times 10^{-3}$ in the cell-unadjusted and cell-adjusted models, respectively, 500 had a p-value $<1 \times 10^{-3}$ according to both models. Adjusting for estimated cell counts appeared to have little effect: at the 500 top sites, the median percentage difference in effect sizes before and after adjustment was $3.4 \%$ and only $26 / 500$ sites changed by $10 \%$ or more. At exactly half of the 500 top sites, adjusting for cell counts reduced the effect size toward the null. At the remaining 250 sites the effect size increased after cell-adjustment. Adjusting for cell counts increased the standard error at 357/500 sites.

The top $500 \mathrm{CpGs}$ (with a p-value $<1 \times 10^{-3}$ according to both the cell-adjusted and cell-unadjusted models) were enriched at $\mathrm{CpG}$ islands $(42.4 \%$ of the top 500 vs $30.9 \%$ in the whole array; $\mathrm{p}<0.0001)$ and first exons $(7.2$ vs $4.7 \%$; $\mathrm{p}=0.0075)$, but under-represented in the open sea $(22.2$ vs $36.2 \%$; $<0.0001)$ or gene bodies ( 25.2 vs $33.3 \% ; \mathrm{p}=0.0001)$.

\section{Secondary models}

With and without adjustment for cell counts, no individual CpG sites were associated with drinking before pregnancy or during the first trimester after FDR correction for multiple testing. One CpG (cg12509712 near $A R S G$ ) was associated with binge drinking, but only after adjustment for cell counts. In addition, one CpG (cg20334115 near PYCR2) was associated with drinking in the second and/or third trimester, but only before adjustment for cell counts. We did not consider these individual sites further due to the lack of consistency between the cell-adjusted and cell-unadjusted models. Results (including effect sizes) for all sites with a p-value $<1 \times 10^{-3}$ are presented in Supplementary File 2, Supplementary Tables 3-10.

\section{Sensitivity analyzes}

There was evidence of heterogeneity at a minority of the top 500 sites associated with sustained maternal alcohol consumption: $100 / 500$ sites had a heterogeneity p-value $<0.05$; $\mathrm{I}^{2}$ was $>40$ at $36 / 500$. After running a random effects meta-analysis at the top 500 , the coefficients for $88 / 500$ changed $>10 \%$ compared with coefficients generated using the fixed effects meta-analysis. Forest plots and results of a leave-one-out analysis (Supplementary File 3) suggested that no single cohort had a disproportionately large influence on the meta-analysis results consistently over all 500 sites.

Of the top 500 sites, 164 were on a published list of possibly problematic probes [51] (Supplementary File 2, Supplementary Tables $1 \& 2$ ). Although these sites may be more likely to contain outliers, cohorts removed outlier values prior to EWAS and used a regression model that is designed to be robust to outliers in the outcome variable.

The direction of effect in our primary model (sustained maternal alcohol consumption during pregnancy) was mostly consistent with results from our other models (Figure 2). As is expected given their similarity, results from the model assessing maternal alcohol consumption in the second/third trimester were particularly consistent with results from the primary model (Spearman's correlation coefficient for regression coefficients at all probes: 0.76 for the cell-adjusted models and 0.74 for the cell-unadjusted models). Results for all sites with a p-value $<1 \times 10^{-3}$ are presented in Supplementary File 2, Supplementary Tables 1-10 and lambdas and number of hits per cohort are provided in Supplementary File 2, Supplementary Table 11.

In ALSPAC, adjusting for cell counts estimated using the adult whole blood reference panel provided similar EWAS results to those obtained when adjusting for cell counts estimated using a cord blood reference panel: at the top 500 sites from the primary meta-analysis, the median percentage change in coefficients in ALSPAC was 7\% (IQR 3-14\%), with 317/500 sites changing less than 10\%. Of the top 500 sites, 219 had a crude p-value $<0.05$ in ALSPAC when using the adult reference and 197/219 (90\%) also had a crude p-value $<0.05$ when using the cord blood reference.

\section{Comparison to associations between FASD \& buccal epithelial DNA methylation in children}

Of $658 \mathrm{CpG}$ sites at which DNA methylation in buccal cells was associated with FASD according to PortalesCasamar et al., 288 (44\%) had the same direction of association (regardless of p-value) in our study of maternal sustained drinking, but none survived correction for multiple testing at 658 sites (FDR-adjusted p-value $<0.05$ ). Of $542 \mathrm{CpG}$ sites within 101 FASD-associated DMRs (identified using DMRcate), 215 (40\%) had the same direction of association in our study of maternal sustained drinking, but none survived correction for multiple testing at 542 sites (FDR-adjusted p-value < 0.05). For all CpGs, the estimated effect sizes reported by Portales-Casamar et al. were larger than those found in our study. Full look-up results for all PACE models are provided in Supplementary File 2, Supplementary Table 12. 


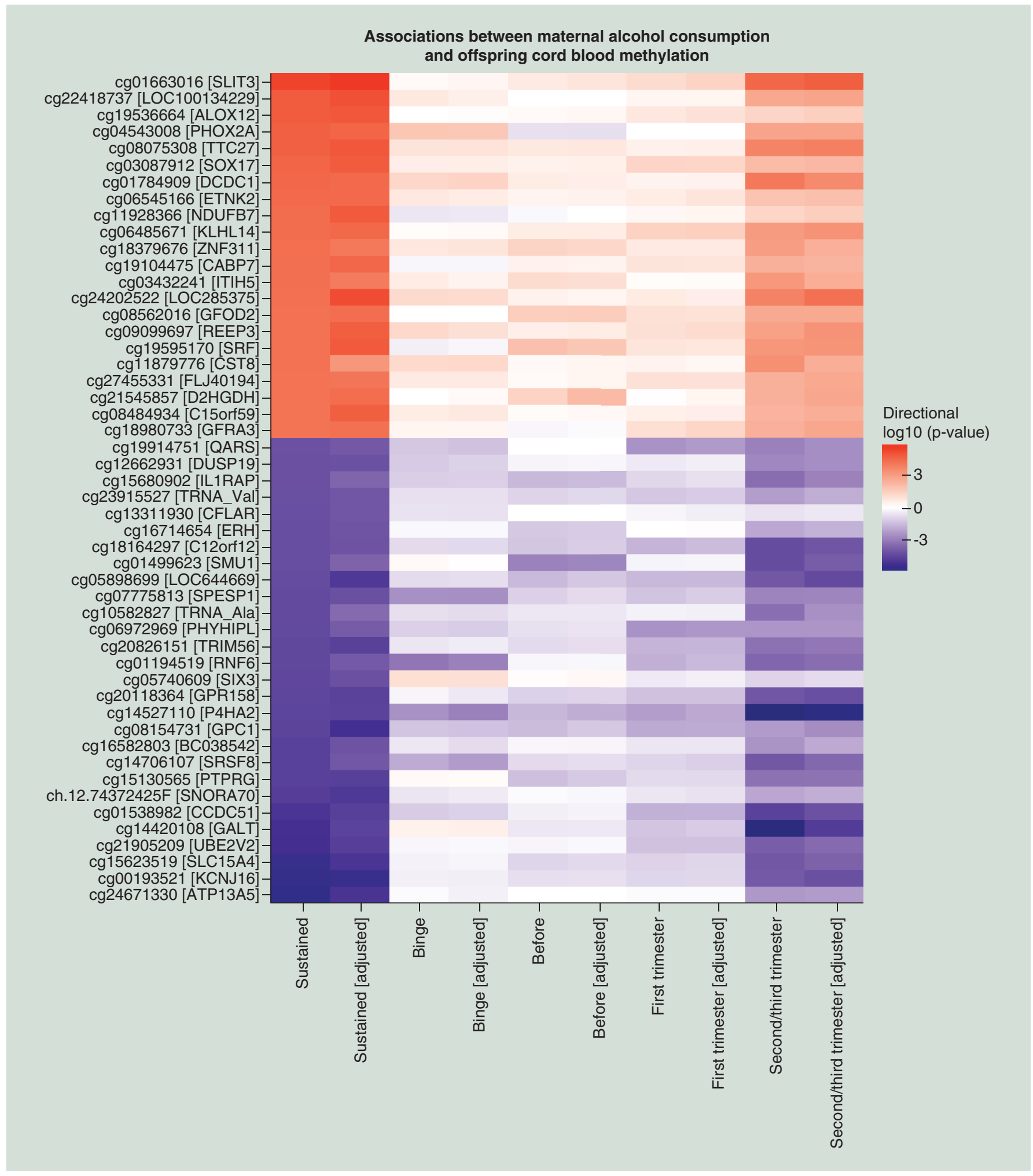

Figure 2. A heatmap to illustrate the direction and strength of association between all investigated alcohol exposures and offspring cord blood DNA methylation. Plotted CpGs are the top $50 \mathrm{CpGs}$ with the smallest p-values in the sustained alcohol consumption single-site EWAS (without adjustment for estimated cell proportions). "Adjusted" denotes models that were adjusted for estimated cell proportions. 
Table 2. Regions identified using the Comb-P method as differentially methylated in association with sustained maternal alcohol consumption.

\begin{tabular}{|c|c|c|c|c|}
\hline Differentially-methylated region (DMR) & CpGs in DMR & Closest gene & $\begin{array}{l}\text { Sidak p-value (unadjusted } \\
\text { for cell counts) }\end{array}$ & $\begin{array}{l}\text { Sidak p-value (adjusted } \\
\text { for cell counts) }\end{array}$ \\
\hline Chr1: $152161237-152162026$ & 7 & $H R N R$ & $1.0 \times 10^{-13}$ & $6.0 \times 10^{-11}$ \\
\hline Chr16: 1583810-1584517 & 8 & IFT140 & $8.7 \times 10^{-13}$ & $5.9 \times 10^{-10}$ \\
\hline Chr6: 49681178-49681775 & 9 & CRISP2 & $9.5 \times 10^{-11}$ & $7.6 \times 10^{-08}$ \\
\hline Chr5:110062343-110062838 & 7 & TMEM232 & $2.1 \times 10^{-10}$ & $2.0 \times 10^{-07}$ \\
\hline Chr17: 6899085-6899759 & 12 & $A L O X 12$ & $1.3 \times 10^{-09}$ & $9.3 \times 10^{-07}$ \\
\hline Chr12: 31271783-31272120 & 4 & $D K F Z p 434 C 0631$ & $8.8 \times 10^{-09}$ & $1.2 \times 10^{-05}$ \\
\hline Chr19: 57741988-57742445 & 10 & AURKC & $1.2 \times 10^{-08}$ & $1.2 \times 10^{-05}$ \\
\hline Chr8: $43131260-43131657$ & 5 & POTEA & $3.8 \times 10^{-07}$ & $4.5 \times 10^{-04}$ \\
\hline Chr5: 8457548-8458090 & 6 & LOC100505738 & $5.5 \times 10^{-07}$ & $4.8 \times 10^{-04}$ \\
\hline Chr13: 76334583-76334867 & 4 & LMO7 & $5.5 \times 10^{-07}$ & $4.8 \times 10^{-04}$ \\
\hline Chr5: 42953543-42953625 & 3 & AK056817 & $9.4 \times 10^{-07}$ & $1.6 \times 10^{-03}$ \\
\hline Chr2: 118594304-118594650 & 3 & $D D X 18$ & $1.2 \times 10^{-06}$ & $7.1 \times 10^{-03}$ \\
\hline Chr3: 145879277-145879711 & 7 & PLOD2 & $1.5 \times 10^{-06}$ & $2.1 \times 10^{-03}$ \\
\hline Chr17: 35423405-35423817 & 4 & AATF & $2.4 \times 10^{-06}$ & $2.6 \times 10^{-03}$ \\
\hline Chr18: 77659572-77659696 & 2 & KCNG2 & $4.1 \times 10^{-06}$ & $4.7 \times 10^{-03}$ \\
\hline Chr15: 69222988-69223369 & 3 & SPESP1 & $4.6 \times 10^{-06}$ & $1.7 \times 10^{-02}$ \\
\hline Chr2: 103236861-103237269 & 2 & SLC9A2 & $6.1 \times 10^{-06}$ & $7.6 \times 10^{-03}$ \\
\hline Chr6: 31846769-31847029 & 10 & SLC44A4 & $6.4 \times 10^{-06}$ & $7.4 \times 10^{-03}$ \\
\hline Chr4: 3516534-3516759 & 4 & LRPAP1 & $9.2 \times 10^{-06}$ & $1.7 \times 10^{-02}$ \\
\hline
\end{tabular}

Comparison to associations between light/moderate drinking \& whole blood DNA methylation in adults

Of $24 \mathrm{CpGs}$ associated (FDR-adjusted $\mathrm{p}<0.05$ ) with adult moderate drinking in the study by Liu et al. [32], one was associated with maternal sustained drinking in our PACE analysis after correction for multiple testing at 24 sites (FDR-adjusted $\mathrm{p}<0.05$ ). At this site, the direction of the effect of maternal drinking on methylation was inverse compared with the effect of own drinking: cg19909613; closest gene TTC35; PACE effect 0.0075 p-value $1.7 \times 10^{-3}$; Liu et al. effect -0.014 p-value $9.2 \times 10^{-7}$. In the cell-adjusted model, this site did not survive FDR correction. None of the three CpG sites that were associated with light drinking according to Liu et al. were associated with maternal sustained drinking in the PACE study. For all CpGs, the estimated effect sizes reported by Liu et al. were larger than those found in our study. Full look-up results for all PACE models are provided in Supplementary File 2, Supplementary Table 13.

\section{DMR analysis}

Using Comb-P to conduct a region-based analysis based on spatial correlation of p-values, we identified 30 and 32 DMRs in the cell-unadjusted and cell-adjusted maternal sustained drinking models, respectively (Sidak-corrected p-value $<0.05$ ). Nineteen regions were differentially methylated according to both models (Table 2; results for all models shown in Supplementary File 2, Supplementary Table 14). However, we found no DMRs when we conducted the region-based analysis using DMRcate. Defining DMRs using a $500 \mathrm{bp}$ (as opposed to $1000 \mathrm{bp}$ ) window did not change our results using either Comb-P or DMRcate.

\section{Blood/brain comparison}

CpGs within the 19 sustained drinking DMRs identified using Comb-P tended to show strong correlations between blood and brain methylation. For example, at the top CpG with the smallest EWAS p-value within the top DMR with the smallest SIDAK-corrected p-value (cg26320663, DMR Chr1:152161237-152162026 an intergenic region on a $\mathrm{CpG}$ island near $H R N R$ ) correlation coefficients between blood and brain ranged from 0.61 in the cerebellum and entorhhinal cortex to 0.66 in the prefrontal cortex (p-values ranging $1.8 \times 10^{-8}$ to $1.5 \times$ $\left.10^{-10}\right)$. All results are presented in Supplementary File 2, Supplementary Table 15. 


\section{Discussion}

We combined data across six pregnancy cohorts to evaluate associations between maternal alcohol consumption during pregnancy and genome-wide DNA methylation in cord blood of offspring. Although we did not find any consistent evidence of association between any category of prenatal alcohol exposure, defined in terms of dose and timing, and single-site methylation, we found some evidence that 19 larger regions of the genome were differentially methylated in association with sustained maternal alcohol consumption throughout pregnancy (i.e., when comparing mothers who drank throughout pregnancy to those who stopped at the beginning or after the first trimester). However, optimal methods for regional analyzes are currently a matter of debate and we were not able to validate this finding using a different region-based method, so we conclude that we have not found any strong evidence for an association.

There are three potential interpretations of the results of our study: light-to-moderate prenatal alcohol exposure does not affect DNA methylation, any association between light-to-moderate prenatal alcohol exposure and DNA methylation is not detectable in cord blood (but could occur in other relevant tissues, e.g. the brain) and a causal association exists, but low statistical power, heterogeneity, measurement error (e.g., targeting the wrong CpG sites), confounding and bias (individually or in combination) prevented us from finding evidence of an association between prenatal alcohol exposure and cord blood DNA methylation.

Interpretation one: no effect of light-to-moderate prenatal alcohol exposure on DNA methylation The first interpretation, that prenatal alcohol exposure does not affect DNA methylation, seems at odds with a body of research in animals that suggests an effect [25-29]. However, it is important to consider alcohol dosage when comparing these studies to our own. Previous population-based studies have shown that women who drink during pregnancy tend to do so at light-to-moderate levels, especially those that drink after pregnancy detection [6]. The dose of prenatal alcohol exposure in our study is likely to be less than that considered by many animal studies. As outlined in the Introduction, evidence of fetal harm caused by light-to-moderate alcohol consumption during pregnancy is inconsistent [7-18]. Therefore, it could be argued that we would not expect light-to-moderate prenatal alcohol exposure to be strongly associated with variation in DNA methylation, either due to low power to detect very small associations, or because there is no causal effect.

Differences in the range of alcohol exposure could also partially explain why we found very limited overlap between our findings and those of a study by Portales-Casamar et al., comparing buccal cells from a small sample of children with and without FASD [31]. At the CpGs that Portales-Casamar et al. identified as associated with FASD, less than half showed the same direction of association with sustained maternal alcohol consumption in our study, and none survived correction for multiple testing. Additionally, the majority of FASD-associated differentially methylated CpGs were in gene bodies, whereas CpGs in gene bodies were significantly underrepresented amongst the top $500 \mathrm{CpGs}$ with the smallest meta-EWAS p-values in our study. The FASD case-control comparison likely covers a larger range of exposure (i.e., cases were exposed to much higher intensity of exposure in utero; controls were likely to have been exposed to less alcohol) than our study of differential prenatal exposure to alcohol in the general population.

However, differences in findings are also likely to arise given the stark methodological differences between the two studies: First, DNA methylation is strongly tissue specific [57], so DNA from buccal cells (Portales-Casamar et al.) and cord blood (our study) are perhaps unlikely to show the same general methylation patterns. Second, DNA methylation is strongly influenced by age [58] and could be affected by many factors in the postnatal environment that are associated with prenatal alcohol exposure (such as maternal education and childhood adversity) - both of these factors are important to consider when interpreting results from our study, where methylation was measured at birth, compared with the study by Portales-Casamar $e t$ al., where participants were around 11 years old. Thirdly, the prospective cohort design of the studies included in our meta-analysis meant that we were able to adjust for measured confounders and participants were sampled from the same populations. Portales-Casamar et al.'s casecontrol study of 11-year-olds is more open to confounding (especially by the postnatal environment, as mentioned above). In particular, the cases and controls differed with respect to important sociodemographic characteristics (namely, ethnicity and being raised by adoptive/foster parents) that have previously been associated with variation in DNA methylation [59-61]. Fourthly, the difference in level of alcohol exposure in both studies means there are different confounding structures, for example, in Portales-Casamar $e t$ al. higher exposure was associated with lower socioeconomic status, whereas, in our study, higher exposure was associated with higher maternal education in all investigated cohorts. 
We also saw limited similarities between our findings and those of a study recently published by Liu et al., which found some evidence of association between DNA methylation and varying levels of alcohol consumption in an adult population [32]. At the CpGs that were associated with light or moderate drinking in Liu et al., we found no strong evidence of an association with prenatal exposure. This lack of overlap might be explained by maternal alcohol consumption affecting own but not fetal DNA methylation. This would provide further support the hypothesis that light-to-moderate prenatal alcohol exposure is not associated with DNA methylation. However, other plausible explanations are that maternal alcohol consumption affects fetal DNA methylation at different CpG sites compared with adult methylation, and/or the lack of overlap is related to differences in population age, definitions, range and duration of exposure, and potential differences in accuracy of self-report in nonpregnant and pregnant adults leading to differences in measurement error.

\section{Interpretation two: the effect of prenatal alcohol consumption on DNA methylation is not} detectable in cord blood

The second possible interpretation of our results is that any association between prenatal alcohol exposure and DNA methylation is not detectable in cord blood. Although we did not find strong evidence of an association in offspring cord blood, this does not exclude the possibility that such an association does exist in a different tissue. For example, brain tissue may be a more appropriate tissue to study because some of the strongest evidence from observational studies suggests an association between prenatal alcohol exposure and impaired neurodevelopmental outcomes [62]. There are obvious ethical issues that preclude collection of brain tissue in population-based studies, however, there are reasons to believe that DNA methylation in blood may be a good surrogate for DNA methylation in brain at some sites. Strong correlations in blood and brain DNA methylation have been found at some CpGs [56,63], including at our top DMR from the Comb-P analysis. Although we were not able to replicate our Comb-P DMRs using a different method (DMRcate), the high correlation between some of these regions in blood and brain mean they may serve as interesting candidate genes for further studies of the role of DNA methylation as a mediator of associations between prenatal alcohol exposure and offspring neurodevelopmental outcomes. Alternatively, the high correlations between blood and brain methylation at these sites might represent a genetic influence on methylation that is also associated with maternal alcohol consumption. Regardless of causality, methylation at these regions might be a useful biomarker for prenatal alcohol exposure [64], thereby serving as a more objective measure than self-report. This possibility would have to be validated and tested in independent datasets.

\section{Interpretation three: the effect of prenatal alcohol consumption on DNA methylation is not} detectable due to methodological limitations

Our study has many strengths, including the use of data from six well-characterized and established cohorts. The prospective data available from these cohorts has allowed us to investigate the timing and strength of exposure, including binge-drinking and exposure by trimester, and to minimize measurement error and recall bias. Previous studies from the PACE consortium have used a similar methodology to prescribe cohort-specific analyzes and to meta-analyze results from several cohorts. These studies identified many, seemingly robust, associations $[22,65-66]$. This suggests that the lack of associations identified in our study is not likely due to poor EWAS or meta-analysis methodology or data. However, the third possible interpretation of our findings is that methodological elements such as statistical power, heterogeneity, confounding and bias prevented us from finding strong evidence of an association between maternal alcohol consumption during pregnancy and offspring cord blood DNA methylation.

Failure to observe associations between maternal alcohol consumption and cord blood DNA methylation could be due to lack of statistical power, particularly if we hypothesize a dose-response association such that low levels of exposure correspond to small methylation differences. As most pregnant women in our cohorts did not drink excessive amounts of alcohol, a large sample size would be required to detect a small epigenetic effect.

A further potential limitation of our study is that there was some intercohort heterogeneity. Sources of this heterogeneity include differences in the range of alcohol exposure, for example, in our main analysis, ALSPAC, GECKO and Generation R have more exposed than unexposed individuals, while the MoBa cohorts have a higher proportion of unexposed individuals. These differences are perhaps expected, because public health advice on drinking during pregnancy has changed over time in different countries. Other potential sources of heterogeneity include differences in how alcohol drinking is measured, confounding structures (we note that in some studies women who drank throughout pregnancy were more likely to be smokers, while in others the opposite was true) and/or EWAS methodological differences such as different methods of normalizing DNA methylation data, coding 
covariates and adjusting for batch. Encouragingly though, our meta-analysis results were not substantially different when we used a random-effects model compared with a fixed-effects model. Furthermore, forest plots and results of a leave-one-out analysis suggested our meta-analysis results were not strongly influenced by differences between studies. In a previous PACE analysis [22], we found that results obtained using raw $\beta$ s were similar to those obtained using $\beta$ s normalized by various methods, which indicates that the method of normalization did not impact the inference drawn from the meta-analysis. Furthermore, nonspurious associations would likely be robust to small differences in methodology between studies.

Measurement error could also reduce statistical power and this may be a particular problem for studies of maternal alcohol consumption: pregnant women may under-report behaviors that are widely thought to be harmful for their baby [67]. Similarly, classification bias may introduce measurement error, for example, harmonizing the definition of binge drinking was particularly difficult because binge drinking was defined differently in different cohorts. As a result, women consuming four drinks on one occasion would be classed as binge drinkers in some cohorts but nonbinge drinkers in others. This reduces the power to detect a true effect.

Cellular heterogeneity in cord blood samples is a further issue [68] that may introduce error in our measure of DNA methylation. Blood samples are highly heterogeneous and although we have attempted to adjust for cellular heterogeneity by including estimated cell proportions in our EWAS models, no suitable cord blood reference was widely available at the time of analysis, so these estimates were based on an adult blood reference panel. When a cord blood reference became available [52], a sensitivity analysis in ALSPAC did not reveal sizable differences between EWAS results adjusted for cell proportions estimated using either the cord or adult reference panel. However, we recognize that there may be a residual influence of cellular heterogeneity that could be biasing the results in either direction. Another factor that could be biasing results is circulating folate levels, which we could not formally evaluate because not all cohorts had the required data. However, there are two reasons why folate is likely to play at most a marginal role in our findings. First, we found low inter-study heterogeneity for most of our CpG sites - if there was a prominent interaction between alcohol (a folate antagonist) and folate (a methyl donor), then we might expect more heterogeneity due to country- and timing-specific differences in folate intake. Secondly, our results were largely null - confounding by folic acid supplementation, which is indirectly associated with alcohol consumption [14,69], is unlikely to be exaggerating estimates of the association between maternal alcohol and offspring methylation.

We also consider that some of our findings may be affected by selection bias: women who are actively trying to get pregnant may abstain from drinking alcohol, but these women were excluded from our analyzes of sustained and binge drinking. However, these women were not excluded from our analyzes of other alcohol exposures (before pregnancy, first trimester, second and third trimester) and our findings were consistently null across all alcohol exposures.

A final potential limitation is that the Illumina HumanMethylation $450 \mathrm{k}$ array covers only $1.7 \%$ of all CpG sites in the human genome, which may not cover potential regions where maternal alcohol consumption is most strongly associated with offspring methylation. In particular, the array is biased toward regulatory regions, whereas the majority of prenatal alcohol-associated regions identified by Portales-Casamar $e t$ al. were in intergenic regions or gene bodies [31]. However, it should be noted that Portales-Casamar et al. also used the Illumina HumanMethylation 450k array, so limitations in array coverage are unlikely to explain differences in findings between these studies. The development of sequence-based approaches methods with better genomic coverage will help overcome this limitation in future studies.

If an association between maternal alcohol consumption and cord blood DNA methylation does exist (e.g., in larger samples and/or different populations with a greater range of alcohol exposure), then future studies should explore whether that association is causal or explained by some of the issues that plague observational studies, such as those discussed above. Confounding by genetics or shared mother-child environmental factors is a particular concern that should be addressed. For example, paternal alcohol consumption could be employed as a 'negative control' that will share the same confounding structure as maternal alcohol consumption but cannot plausibly affect offspring DNA methylation through a direct causal intrauterine mechanism [70]. Techniques such as two-step Mendelian randomization [71] could also be applied to explore the causal effect of prenatal alcohol exposure on DNA methylation and the causal effect of DNA methylation on offspring outcomes. Even if the association is not causal, newborn blood DNA methylation might capture both genetic and environmental influences of maternal alcohol consumption, which could be useful, both clinically and in research, as a biomarker of exposure and/or a useful predictor of offspring outcomes. 


\section{Conclusion}

In this multicohort study, we found no evidence that maternal alcohol consumption during pregnancy is associated with offspring cord blood DNA methylation in the general population. However, it is possible that exploration of a combination of different tissues, higher doses and different timings of exposure, as well as a more global assessment of genomic DNA methylation, might show evidence of association. We therefore recommend caution when interpreting the present null findings and encourage further investigations.

\section{Summary points}

- It is well known that heavy alcohol consumption during pregnancy can cause Fetal Alcohol Spectrum Disorders (FASD), but evidence is lacking or mixed on the effects of light-to-moderate drinking.

- Whether there is a causal association between light-to-moderate drinking in pregnancy and children's health outcomes is an important question, and identifying a possible biological pathway showing effects at birth would be a step toward providing an answer.

- Some evidence from animal studies suggests that epigenetic mechanisms, such as DNA methylation, might mediate associations between prenatal alcohol exposure and health outcomes.

- In the Pregnancy and Childhood Epigenetics (PACE) consortium, we meta-analyzed associations between maternal drinking in pregnancy and genome-wide cord blood DNA methylation across six population-based birth cohorts, looking at sustained drinking throughout pregnancy, binge drinking and time-specific exposures.

- Our main analysis of sustained maternal drinking included 3075 mother-child pairs, of which 1147 (37.3\%) mothers consumed alcohol both before and throughout pregnancy and the remaining 1928 mothers consumed alcohol before pregnancy/during the first trimester but not during the second and/or third trimester (i.e., they stopped drinking after pregnancy detection).

- We found no strong evidence of association between prenatal alcohol exposure and cord blood DNA methylation at either individual CpGs or across larger regions of the genome.

- We consider three potential interpretations of the results of our study: light-to-moderate prenatal alcohol exposure does not affect DNA methylation, any association between light-to-moderate prenatal alcohol exposure and DNA methylation is not detectable in cord blood (but could occur in other relevant tissues, e.g., the brain) and a causal association is there, but low statistical power, heterogeneity, measurement error (e.g., targeting the wrong $\mathrm{CpG}$ sites), confounding and bias (individually or in combination) prevented us from finding evidence of an association between prenatal alcohol exposure and cord blood DNA methylation.

- It is possible that exploration of a combination of different tissues, higher doses and different timings of exposure, as well as a more global assessment of genomic DNA methylation, might show evidence of association between maternal alcohol consumption and cord blood DNA methylation in the general population.

- We recommend caution when interpreting our null findings and encourage further investigations.

Financial \& competing interests disclosure

The ALSPAC cohort was supported by the Wellcome Trust (WT088806). The Project Viva cohort is funded by NIH grants R01 HL111108, R01 NR013945, and R01 HD034568. The authors have no other relevant affiliations or financial involvement with any organization or entity with a financial interest in or financial conflict with the subject matter or materials discussed in the manuscript apart from those disclosed.

No writing assistance was utilized in the production of this manuscript.

\section{Ethical conduct of research}

The authors state that they have obtained appropriate institutional review board approval or have followed the principles outlined in the Declaration of Helsinki for all human or animal experimental investigations. In addition, for investigations involving human subjects, informed consent has been obtained from the participants involved.

\section{Open access}

This article is distributed under the terms ofthe Creative Commons Attribution License 4.0 which permits any use, distribution, and reproduction in any medium, provided the original author(s) and the source are credited. To view a copy of the license, visit http://creativecommons.org/licenses/by/4.0/ 
Supplementary data

To view the supplementary data that accompany this paper please visit the journal website at: www.futuremedicine.com/doi/full/10.2217/epi-2017-0095

\section{References}

Papers of special note have been highlighted as: $\bullet$ of interest; $\bullet \bullet$ of considerable interest

1. Jones KL, Smith DW. Recognition of the fetal alcohol syndrome in early infancy. Lancet 302(7836), 999-1001 (1973).

2. Abel EL. Fetal Alcohol Abuse Syndrome. Springer Science and Business Media, NY, USA (1998).

3. Sokol RJ, Delaney-Black V, Nordstrom B. Fetal alcohol spectrum disorder. JAMA 290(22), 2996-2999 (2003).

4. Bonthius DJ, Goodlett CR, West JR. Blood alcohol concentration and severity of microencephaly in neonatal rats depend on the pattern of alcohol administration. Alcohol 5(3), 209-214 (1988).

5. Goodlett CR, Horn KH, Zhou FC. Alcohol teratogenesis: mechanisms of damage and strategies for intervention. Exp. Biol. Med. (Maywood) 230(6), 394-406 (2005).

6. O’Keeffe LM, Kearney PM, McCarthy FP et al. Prevalence and predictors of alcohol use during pregnancy: findings from international multicentre cohort studies. BMJ Open 5(7), e006323 (2015).

- Provides interesting and much-needed evidence on the prevalence of alcohol use during pregnancy. It shows that, in the general population, most women who drink during pregnancy do so at light-to-moderate levels.

7. Henderson J, Gray R, Brocklehurst P. Systematic review of effects of low-moderate prenatal alcohol exposure on pregnancy outcome. BJOG An. Int. J. Obstet. Gynaecol. 114(3), 243-252 (2007).

8. McCarthy FP, O'Keeffe LM, Khashan AS et al. Association between maternal alcohol consumption in early pregnancy and pregnancy outcomes. Obstet. Gynecol. 122(4), 830-837 (2013).

9. Patra J, Bakker R, Irving H, Jaddoe V, Malini S, Rehm J. Dose-response relationship between alcohol consumption before and during pregnancy and the risks of low birthweight, preterm birth and small for gestational age (SGA)-a systematic review and meta-analyses. BJOG An. Int. J. Obstet. Gynaecol. 118(12), 1411-1421 (2011).

10. O'Keeffe LM, Greene RA, Kearney PM. The effect of moderate gestational alcohol consumption during pregnancy on speech and language outcomes in children: a systematic review. Syst. Rev. 3(1), 1 (2014).

11. Gaskins AJ, Rich-Edwards JW, Williams PL, Toth TL, Missmer SA, Chavarro JE. Prepregnancy low to moderate alcohol intake is not associated with risk of spontaneous abortion or stillbirth. J. Nutr. 146(4), 799-805 (2016).

12. Flak AL, Su S, Bertrand J, Denny CH, Kesmodel US, Cogswell ME. The association of mild, moderate, and binge prenatal alcohol exposure and child neuropsychological outcomes: a meta-analysis. Alcohol. Clin. Exp. Res. 38(1), 214-226 (2014).

13. Bay B, Kesmodel US. Prenatal alcohol exposure - a systematic review of the effects on child motor function. Acta Obstet. Gynecol. Scand. 90(3), 210-226 (2011).

14. Zuccolo L, DeRoo LA, Wills AK et al. Pre-conception and prenatal alcohol exposure from mothers and fathers drinking and head circumference: results from the Norwegian Mother-Child Study (MoBa). Sci. Rep. 7, 39535 (2016).

15. Mamluk L, Edwards HB, Savović J et al. Low alcohol consumption and pregnancy and childhood outcomes: time to change guidelines indicating apparently 'safe' levels of alcohol during pregnancy? A systematic review and meta-analyses. BMJ Open 7(7), e015410 (2017).

- This is a comprehensive review of all the available studies of light alcohol consumption and pregnancy and childhood outcomes since the 1950s. It finds surprisingly limited evidence that light drinking during pregnancy poses any risk to the baby, but there was a weak association with preterm delivery and being small-for-gestational-age.

16. Lewis SJ, Zuccolo L, Davey Smith G et al. Fetal alcohol exposure and IQ at age 8: evidence from a population-based birth-cohort study. PLoS ONE 7(11), e49407 (2012).

17. Zuccolo L, Lewis SJ, Davey Smith G et al. Prenatal alcohol exposure and offspring cognition and school performance. A 'Mendelian randomization' natural experiment. Int. J. Epidemiol. 42(5), 1358-1370 (2013).

18. Murray J, Burgess S, Zuccolo L, Hickman M, Gray R, Lewis SJ. Moderate alcohol drinking in pregnancy increases risk for children's persistent conduct problems: Causal effects in a Mendelian randomisation study. J. Child Psychol. Psychiatry Allied Discip. 57(5), 575-584 (2016).

19. Gray R. Low-to-moderate alcohol consumption during pregnancy and child development - moving beyond observational studies. BJOG 120(9), 1039-1041 (2013).

20. Niclasen J. Drinking or not drinking in pregnancy: the multiplicity of confounding influences. Alcohol Alcohol. 49(3), 349-355 (2014).

21. Department of Health. UK Chief Medical Officers' Alcohol Guidelines Review: summary of the proposed new guidelines (2016). www.gov.uk/government/uploads/system/uploads/attachment_data/file/489795/summary.pdf

22. Joubert BR, Felix JF, Yousefi P et al. DNA methylation in newborns and maternal smoking in pregnancy: Genome-wide Consortium meta-analysis. Am. J. Hum. Genet. 98(4), 680-696 (2016). 
-• This previous study from the PACE consortium identified many strong associations between maternal smoking throughout pregnancy and DNA methylation in offspring cord blood at birth and peripheral blood later in childhood.

23. Richmond RC, Simpkin AJ, Woodward G et al. Prenatal exposure to maternal smoking and offspring DNA methylation across the lifecourse: findings from the Avon Longitudinal Study of Parents and Children (ALSPAC). Hum. Mol. Genet. 24(8), 2201-2217 (2015).

24. Küpers LK, $\mathrm{Xu}$ X, Jankipersadsing SA et al. DNA methylation mediates the effect of maternal smoking during pregnancy on birthweight of the offspring. Int. J. Epidemiol. 44(4), 1224-1237 (2015).

25. Halsted $\mathrm{CH}$, Medici V. Aberrant hepatic methionine metabolism and gene methylation in the pathogenesis and treatment of alcoholic steatohepatitis. Int. J. Hepatol. 2012, 959746 (2012).

26. Hutson JR, Stade B, Lehotay DC, Collier CP, Kapur BM. Folic acid transport to the human fetus is decreased in pregnancies with chronic alcohol exposure. PLoS ONE 7(5), e38057 (2012).

27. Stouder C, Somm E, Paoloni-Giacobino A. Prenatal exposure to ethanol: a specific effect on the H19 gene in sperm. Reprod. Toxicol. 31(4), 507-512 (2011).

28. Perkins A, Lehmann C, Lawrence RC, Kelly SJ. Alcohol exposure during development: Impact on the epigenome. Int. J. Dev. Neurosci. 31(6), 391-397 (2013).

29. Laufer BI, Chater-Diehl EJ, Kapalanga J, Singh SM. Long-term alterations to DNA methylation as a biomarker of prenatal alcohol exposure: From mouse models to human children with fetal alcohol spectrum disorders. Alcohol 60, 67-75 (2017).

30. Khalid O, Kim JJ, Kim H-S et al. Gene expression signatures affected by alcohol-induced DNA methylomic deregulation in human embryonic stem cells. Stem Cell Res. 12(3), 791-806 (2014).

31. Portales-Casamar E, Lussier AA, Jones MJ et al. DNA methylation signature of human fetal alcohol spectrum disorder. Epigenetics Chromatin. 9, 25 (2016).

-• This is one of the only previously published studies of prenatal alcohol exposure and DNA methylation in humans. The authors found several regions differentially methylated in buccal cells from children with and without FASD. In the current paper, we discuss the many possible reasons for very limited overlap between these results and our own.

32. Liu C, Marioni RE, Hedman AK et al. A DNA methylation biomarker of alcohol consumption. Mol. Psychiatry. doi:10.1038/mp.2016.192 (2016) (Epub ahead of print).

-• This meta-analysis from the CHARGE + consortium identified many CPG sites where DNA methylation is associated with alcohol consumption in adults. We performed a look-up of their results within our dataset and found very limited overlap. Potential reasons are discussed in the current paper.

33. Sandoval J, Heyn HA, Moran S et al. Validation of a DNA methylation microarray for 450,000 CpG sites in the human genome. Epigenetics 6(6), 692-702 (2011).

34. Relton CL, Gaunt T, McArdle W et al. Data Resource Profile: Accessible Resource for Integrated Epigenomic Studies (ARIES). Int. J. Epidemiol. 44(4), 1181-1190 (2015).

35. Boyd A, Golding J, Macleod J et al. Cohort Profile: the 'children of the 90s' - the index offspring of the Avon Longitudinal Study of Parents and Children. Int. J. Epidemiol. 42(1), 111-127 (2013).

36. Fraser A, Macdonald-Wallis C, Tilling K et al. Cohort Profile: the Avon Longitudinal Study of Parents and Children: ALSPAC mothers cohort. Int. J. Epidemiol. 42(1), 97-110 (2013).

37. L'Abee C, Sauer PJ, Damen M, Rake J-P, Cats H, Stolk RP. Cohort Profile: the GECKO Drenthe study, overweight programming during early childhood. Int. J. Epidemiol. 37(3), 486-489 (2008).

38. Kruithof CJ, Kooijman MN, van Duijn CM et al. The Generation R Study: Biobank update 2015. Eur. J. Epidemiol. 29(12), 911-27 (2014).

39. Kooijman MN, Kruithof CJ, van Duijn CM et al. The Generation R Study: design and cohort update 2017. Eur. J. Epidemiol. 31(12), 1243-1264 (2016).

40. Magnus P, Irgens LM, Haug K et al. Cohort profile: the Norwegian Mother and Child Cohort Study (MoBa). Int. J. Epidemiol. 35(5), 1146-50 (2006).

41. Rønningen KS, Paltiel L, Meltzer HM et al. The biobank of the Norwegian Mother and Child Cohort Study: a resource for the next 100 years. Eur. J. Epidemiol. 21(8), 619-25 (2006).

42. Oken E, Baccarelli AA, Gold DR et al. Cohort profile: project viva. Int. J. Epidemiol. 44(1), 37-48 (2015).

43. Houseman EA, Accomando WP, Koestler DC et al. DNA methylation arrays as surrogate measures of cell mixture distribution. BMC Bioinformatics 13(1), 86 (2012).

44. Reinius LE, Acevedo N, Joerink M et al. Differential DNA methylation in purified human blood cells: implications for cell lineage and studies on disease susceptibility. PLoS ONE 7(7), e41361 (2012).

45. Tukey JW. Exploratory data analysis. Addison-Wesley Publishing Company Reading, Mass. Biometric Journal 23(4), 413-414 (1981).

46. Venables W, Ripley B. Modern Applied Statistics with S. Fourth. Springer, NY, USA (2002). 
47. Willer CJ, Li Y, Abecasis GR. METAL: fast and efficient meta-analysis of genomewide association scans. Bioinformatics 26(17), 2190-2191 (2010).

48. Benjamini Y, Hochberg Y. Controlling the false discovery rate: a practical and powerful approach to multiple testing. J. R. Stat. Soc. Ser. B. 57(1), 289-300 (1995).

49. Hansen K. IlluminaHumanMethylation450kanno.ilmn12.hg19: annotation for Illumina's 450k methylation arrays. Bioconductor doi:10.18129/B9.bioc.IlluminaHumanMethylation450kanno.ilmn12.hg19 (2016) (Epub ahead of print).

50. Viechtbauer W. Conducting meta-analyses in R with the metafor Package. J. Stat. Softw. 36(3), 1-48 (2010).

51. Naeem $\mathrm{H}$, Wong $\mathrm{N}$, Chatterton $\mathrm{Z}$ et al. Reducing the risk of false discovery enabling identification of biologically significant genome-wide methylation status using the HumanMethylation450 array. BMC Genomics 15(1), 51 (2014).

52. Andrews S, Bakulski K. FlowSorted.CordBlood.450k: Illumina 450k data on sorted cord blood cells. Bioconductor doi:10.18129/B9.bioc.FlowSorted.CordBlood.450k (2016) (Epub ahead of print).

53. Pedersen BS, Schwartz DA, Yang IV, Kechris KJ. Comb-p: software for combining, analyzing, grouping and correcting spatially correlated P-values. Bioinformatics 28(22), 2986-2988 (2012).

54. Peters TJ, Buckley MJ, Statham AL et al. De novo identification of differentially methylated regions in the human genome. Epigenetics Chromatin 8(1), 6 (2015).

55. Complex Disease Epigenetics Group. Blood Brain DNA Methylation Comparison Tool. http://epigenetics.iop.kcl.ac.uk/bloodbrain/

56. Hannon E, Lunnon K, Schalkwyk L, Mill J. Interindividual methylomic variation across blood, cortex, and cerebellum: implications for epigenetic studies of neurological and neuropsychiatric phenotypes. Epigenetics 10(11), 1024-1032 (2015).

57. Lokk K, Modhukur V, Rajashekar B et al. DNA methylome profiling of human tissues identifies global and tissue-specific methylation patterns. Genome Biol. 15(4), r54 (2014).

58. Horvath S. DNA methylation age of human tissues and cell types. Genome Biol. 14(10), R115 (2013).

59. Fraser HB, Lam LL, Neumann SM, Kobor MS. Population-specificity of human DNA methylation. Genome Biol. 13(2), R8 (2012).

60. Essex MJ, Boyce WT, Hertzman C et al. Epigenetic vestiges of early developmental adversity: childhood stress exposure and DNA methylation in adolescence. Child Dev. 84(1), 58-75 (2013).

61. Roth TL, Lubin FD, Funk AJ, Sweatt JD. Lasting epigenetic influence of early-life adversity on the bdnf gene. Biol. Psychiatry 65(9), 760-769 (2009).

62. Birney E, Davey Smith G, Greally JM. Epigenome-wide association studies and the interpretation of disease -omics. PLoS Genet. 12(6), e1006105 (2016).

63. Walton E, Hass J, Liu J et al. Correspondence of DNA methylation between blood and brain tissue and its application to schizophrenia research. Schizophr. Bull. 42(2), 406-414 (2016).

64. Relton CL, Hartwig FP, Davey Smith G. From stem cells to the law courts: DNA methylation, the forensic epigenome and the possibility of a biosocial archive. Int. J. Epidemiol. 44(4), 1083-1093 (2015).

65. Gruzieva O, Xu C-J, Breton CV et al. Epigenome-wide meta-analysis of methylation in children related to prenatal NO2 air pollution exposure. Environ. Health Perspect. 125(1), 104-110 (2017).

66. Sharp GC, Salas LA, Monnereau C et al. Maternal BMI at the start of pregnancy and offspring epigenome-wide DNA methylation: findings from the pregnancy and childhood epigenetics (PACE) consortium. Hum. Mol. Genet. 26(20), 4067-4085 (2017).

-. This previous study from the PACE consortium found weak associations between maternal body mass index at the start of pregnancy and offspring DNA methylation. After application of causal inference techniques, we found stronger evidence that the majority of these associations were better explained by shared genetic and/or environmental factors than a causal intrauterine effect.

67. Jacobson SW, Jacobson JL, Sokol RJ, Martier SS, Ager JW, Kaplan MG. Maternal recall of alcohol, cocaine, and marijuana use during pregnancy. Neurotoxicol. Teratol. 13(5), 535-540 (1991).

68. Jaffe AE, Irizarry RA. Accounting for cellular heterogeneity is critical in epigenome-wide association studies. Genome Biol. 15(2), R31 (2014).

69. Cueto HT, Riis AH, Hatch EE, Wise LA, Rothman KJ, Mikkelsen EM. Predictors of preconceptional folic acid or multivitamin supplement use: a cross-sectional study of Danish pregnancy planners. Clin. Epidemiol. 4, 259-265 (2012).

70. Richmond RC, Al-Amin A, Davey Smith G, Relton CL. Approaches for drawing causal inferences from epidemiological birth cohorts: a review. Early Hum. Dev. 90(11), 769-780 (2014).

71. Relton CL, Davey Smith G. Two-step epigenetic Mendelian randomization: a strategy for establishing the causal role of epigenetic processes in pathways to disease. Int. J. Epidemiol. 41(1), 161-176 (2012). 